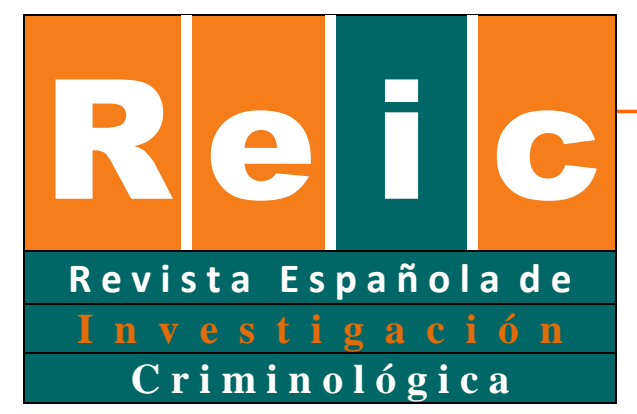

León \& Aizpurúa

\title{
Percepciones y sentimientos hacia las víctimas: Resultados de un experimento split-ballot
}

\section{Perceptions and feelings towards victims: The results of a split- ballot experiment}

\author{
Carmen María León ${ }^{1}$ (iD) \\ Centro de Investigación en Criminología, Universidad de Castilla-La Mancha
}

Eva Aizpurúa (iD

Center for Social and Behavioral Research, University of Northern Iowa

\section{RESUMEN}

El diseño del cuestionario es una parte esencial de la investigación cuantitativa. En este trabajo se exponen los resultados de un estudio sobre actitudes hacia las víctimas en el que se incluyó un experimento. Su primer objetivo consistió en analizar las tipologías de víctimas en las que piensan los encuestados cuando son preguntados, de forma abstracta, por víctimas de delitos. En segundo lugar, el propósito del experimento fue comprobar cómo afecta el orden de presentación de las respuestas en una pregunta sobre los sentimientos que suscitan las víctimas. Al tratarse de un cuestionario auto-administrado, hipotetizamos que los participantes $(\mathrm{N}=100)$ tenderían a elegir las primeras opciones de respuesta (efecto primacía). Para comprobarlo se recurrió a un diseño split-ballot. Los resultados muestran que los encuestados piensan en las víctimas de aquellos delitos que (1) se producen con mayor frecuencia y (2) son más graves. En cambio, no permiten confirmar el efecto primacía en este experimento.

\footnotetext{
${ }^{1}$ La correspondencia debe dirigirse a: Carmen María León. Facultad de Derecho de Albacete (Universidad de Castilla-La Mancha). Plaza de la Universidad, 1. 02071 Albacete. Carmen.Leon@uclm.es
}

Revista Española de Investigación Criminológica

Artículo 6, Número 16 (2018)

https://doi.org/10.46381/reic.v16i0.158

Www.criminologia.net

ISSN: 1696-9219 
Palabras clave: metodología de encuestas, diseño experimental, efectos del orden de las respuestas, actitudes hacia las víctimas.

\begin{abstract}
Questionnaire design is an essential part of quantitative research. In this paper, we present the results of a study on attitudes towards victims, in which an experiment was included. The first objective of this work was to analyze the types of victims respondents think about when they are asked, in abstract form, about victims of crimes. Secondly, the purpose of the experiment was to verify how the order in which answers are presented affects the feelings that victims elicit. As a self-administered questionnaire, we hypothesized that the participants $(\mathrm{N}=100)$ would tend to choose the first response options (primacy effect). For this, a splitballot design was used. The results showed that respondents think about the victims of those crimes that (1) occur most frequently and (2) are the most serious. The results did not confirm the primacy effect in this experiment.
\end{abstract}

Keywords: survey methodology, experimental design, response order effects, attitudes towards victims.

\title{
1. Introducción
}

Las actitudes hacia el castigo de quienes trasgreden las normas son un ámbito de gran interés criminológico. En la actualidad, esta línea de investigación se encuentra ampliamente expandida y cuenta con numerosos estudios tanto a nivel internacional como en el contexto español que permiten conocer las actitudes de la población hacia el sistema de justicia penal. Sin embargo, no se puede afirmar lo mismo con respecto al campo de las actitudes públicas hacia las víctimas de delitos. Tradicionalmente, los estudios que han analizado las actitudes hacia el castigo se han centrado principalmente en el delito y en la figura del agresor. En este sentido, han examinado cómo influye el tipo de delito, el sexo, la raza y el estatus social del delincuente en las decisiones tomadas por la ciudadanía acerca del castigo que debería serle impuesto (Applegate, Cullen, Link, Richards, \& Lanza-Kaduce, 1996; Baz, Aizpurúa, \& Fernández, 2015; DeSantis \& Kayson, 1997; Simonson, 2011). Más recientemente, también han sido analizadas las variables relativas a las víctimas que inciden en las demandas de castigo de la ciudadanía. Así, si bien los resultados no han sido del todo concluyentes, la 2

Revista Española de Investigación Criminológica

Artículo 6, Número 16 (2018)

https://doi.org/10.46381/reic.v16i0.158

Www.criminologia.net

ISSN: 1696-9219 
evidencia muestra que la población demanda medidas más punitivas en aquellos casos en los que las víctimas son mujeres y niños (Mazzela \& Feingold, 1994; Pickett, Mancini, \& Mears, 2013), el daño causado es mayor (Brocke, Goldenitz, Holling, \& Bilsky, 2004; Hills \& Thomson, 1999) y las víctimas despiertan mayor simpatía (Myers \& Greene, 2004).

Sin embargo, y a pesar de que los estudios realizados en el ámbito de las actitudes hacia el castigo han incorporado la figura de la víctima para determinar cómo influye en las demandas de castigo, son escasos los estudios que han analizado específicamente las actitudes y percepciones hacia las víctimas de delitos, especialmente en el contexto español. Además, la mayor parte de los estudios llevados a cabo en este ámbito se han limitado a analizar las actitudes hacia las víctimas de un delito muy concreto como es la violación. Estos estudios han puesto de relieve la tendencia generalizada a culpabilizar a las víctimas por lo ocurrido (Cameron \& Stritzke, 2003; Niemi \& Young, 2014, 2016; Rini, 2015; Sjöberg, \& Sarwar, 2017) y han señalado la necesidad de analizar determinados elementos, como el tipo de delitos, las creencias estereotipadas sobre las víctimas o las circunstancias en las que ocurren los hechos, en el estudio de las actitudes hacia las víctimas.

Por ello, y con la finalidad de contribuir a este campo de investigación, el propósito de este artículo es analizar, por un lado, el tipo de delitos en los que piensan los encuestados al ser preguntados por la víctima de un delito en abstracto y, por otro lado, los sentimientos que suscitan las víctimas. Además, dentro de este segundo propósito se llevará a cabo un experimento para determinar cómo afecta el orden de presentación de las alternativas de respuesta en un cuestionario auto-administrado.

\subsection{El uso de preguntas abstractas en el ámbito de las actitudes hacia el castigo}

Debido a la ausencia de estudios acerca de las actitudes hacia las víctimas de delitos, parece necesario recurrir al campo de las actitudes hacia el castigo para conocer cómo podría afectar la formulación abstracta de preguntas en relación con este ámbito. En su inicio, los trabajos que exploraron las actitudes punitivas emplearon cuestionarios elementales compuestos por preguntas generales ("In general, do you think the courts in this area deal too harshly or not harshly enough with criminals?"). Estos estudios tendían a mostrar una ciudadanía punitiva,

Revista Española de Investigación Criminológica

Artículo 6, Número 16 (2018)

https://doi.org/10.46381/reic.v16i0.158

Www.criminologia.net

ISSN: 1696-9219 
en parte porque la imprecisión de las preguntas provocaba que la población pensase en los casos más graves al emitir sus respuestas, expresando así actitudes más punitivas (Hough \& Roberts, 2002). Estos hallazgos constataron que las actitudes públicas son demasiado complejas como para ser descritas y sintetizadas a través de preguntas generales (Jerre, 2014) $\mathrm{y}$, a partir de este momento, se produjo una modificación sustancial de los cuestionarios iniciales, incorporando a las preguntas tradicionales los denominados casos-escenario (vignettes). Estos casos describen situaciones y personas concretas, previniendo que los encuestados basen sus respuestas en estereotipos (Kury \& Ferdinand, 1999; Steinberg \& Piquero, 2010) y proporcionando una imagen menos punitiva (Roberts, Stalans, Indemaur, \& Hough, 2003).

A la vista de estos hallazgos, se espera que los encuestados, al responder a preguntas generales sobre víctimas, tiendan a pensar, al igual que ocurre en el ámbito de las actitudes punitivas, en aquellos casos más graves.

\subsection{Sentimientos que suscitan las víctimas de violencia}

El ámbito de los sentimientos que suscitan las víctimas de violencia ha sido tradicionalmente estudiado desde la psicología. La mayor parte de los estudios dedicados a este fin han utilizado como metodología el visionado de escenas violentas para evaluar las posiciones de los espectadores respecto a la víctima destinataria de esa violencia. Estos estudios han concluido que, para construir sus posiciones ante la víctima, los espectadores procesan la representación que se hace de ellas, evalúan moralmente las situaciones en las que ocurre la violencia y procesan sus propias experiencias personales (Fernández \& Revilla, 2016). Además, han identificado dos posiciones enfrentadas: el acercamiento y el distanciamiento con la víctima. El distanciamiento reduce la posibilidad de las imágenes de emocionar al espectador y, en cierto modo, descuida la humanidad de las víctimas. Pero, principalmente, conlleva una actitud de normalización en la que el espectador no se siente concernido por los sucesos. El distanciamiento puede presentarse a través de diferentes modalidades como la deshumanización, la culpabilización o la incomprensión de las víctimas. Por otro lado, el acercamiento supone una reducción de la distancia psicológica con la víctima. Esta posición 
humaniza el sufrimiento del otro y lo comprende, genera sentimientos de preocupación, compasión o pesadumbre por ellos y reflexiona sobre las causas de los hechos y las maneras de evitarlos (Fernández \& Revilla, 2016).

En consecuencia, parece que los sentimientos que susciten las víctimas de delitos dependerán de diversos factores como la representación que los encuestados hagan de ellas, de la evaluación moral que efectúen de la situación en la que se ha producido el delito, de sus propias experiencias personales de victimización e incluso de la visión estereotipada que tengan acerca de las víctimas, entre otros aspectos.

\subsection{Efectos en el orden de presentación de las alternativas de respuesta}

Investigaciones previas han demostrado que el orden de presentación de las alternativas de respuesta puede afectar a su probabilidad de ser seleccionadas (Krosnick \& Alwin, 1987; Quinn \& Belson, 1969). La teoría sobre los efectos de posición serial señala la existencia de dos fenómenos diferentes: primacía y recencia. El primero de ellos tiene lugar cuando las alternativas de respuesta se presentan de forma visual, como ocurre en las encuestas autoadministradas. Este fenómeno se produce cuando las opciones de respuesta que se presentan primero tienen una mayor probabilidad de ser elegidas. Por el contrario, el efecto de recencia implica la mayor elección de las últimas categorías y es habitual en las encuestas telefónicas y personales donde las opciones de respuesta se presentan oralmente (Holbrook, Krosnick, Moore, \& Tourangeau, 2007). Este último efecto vendría explicado por el sistema de memoria a corto plazo ya que, en el momento de evaluar todas las alternativas, los ítems presentados más recientemente serán recordados con mayor facilidad (Krosnick \& Alwin, 1987).

\subsubsection{Investigación previa: efecto primacía}

El efecto primacía ha sido documentado mayoritariamente en cuestionarios autoadministrados (Bishop, Hipplet, Schwarz, \& Strack, 1988; Israel \& Taylor, 1990; Krosnick \& Alwin, 1987; Schwarz \& Bless, 1992). Sin embargo, no todos los estudios que han tratado de documentar este efecto lo han conseguido con éxito. En este sentido, si bien algunos 
trabajos sí han constatado el efecto primacía entre las categorías de respuesta (Bishop et al., 1988; Israel \& Taylor, 1990; Krosnick \& Alwin, 1987; Schwarz \& Bless, 1992), otros no lo han hecho (Chang \& Krosnick, 2009) o sólo lo han encontrado en determinadas preguntas (Galesic, Tourangeau, Couper, \& Conrad, 2008; Stern, Dillman, \& Smyth, 2007).

Desde el ámbito de la psicología se han propuesto principalmente dos explicaciones a este fenómeno. En primer lugar, se ha sostenido que la mayor probabilidad de seleccionar aquellas respuestas presentadas al inicio de la lista podría deberse a que los encuestados emplean un mayor tiempo en procesar y analizar estas alternativas. Además, este procesamiento más exhaustivo de las primeras categorías afectaría también a las alternativas posteriores, que serían analizadas menos cuidadosamente y estimularían menos justificaciones por las que ser elegidas. Por otro lado, e íntimamente relacionado con lo anterior, se ha afirmado que ese procesamiento más exhaustivo de las primeras categorías de respuesta derivaría en la fatiga del encuestado. De tal modo que el procesamiento de las alternativas posteriores sería significativamente más reducido, pudiendo incluso hacer que los encuestados no se molesten en leer las últimas alternativas de respuesta, seleccionando directamente los ítems situados al inicio de la lista, siempre que se consideren razonables (Galesic et al., 2008; Krosnick, 1991; Krosnick \& Alwin, 1987; Tourangeau, 1984).

Además, también se han analizado aquellas características de los encuestados que podrían favorecer el efecto primacía. En este sentido, Stern y sus colaboradores (2007), si bien encontraron que no en todas las preguntas se producía el efecto primacía, hallaron que cuando este fenómeno ocurría, se producía con independencia de la edad, del nivel educativo y del sexo de los participantes. No obstante, y como muestra la literatura acumulada, estos resultados no son consistentes. Aunque Krosnick y Alwin (1987) encontraron que los sujetos con niveles educativos más bajos eran más susceptibles al efecto primacía, estudios posteriores no han permitido confirmar estas averiguaciones (Ayidiya \& McClendon, 1990). De igual forma, Knaüper (1999) encontró que, si bien la edad parece ser un poderoso predictor de los efectos de orden de respuesta, era necesario ampliar las investigaciones incluyendo encuestados de mayor edad. En cuanto al sexo, han sido menos numerosos los estudios que han examinado las diferencias en los efectos de respuesta entre hombres y 
mujeres. Además, los resultados no han sido consistentes y muestran que es necesario seguir indagando en este ámbito.

\section{Objetivos e hipótesis}

En este trabajo se plantean dos objetivos principales:

El primero de ellos consiste en analizar las tipologías de víctimas en las que piensan los encuestados cuando son preguntados, de manera abstracta, por la víctima de un delito, para compararlas, después, con las estadísticas delictivas oficiales y de auto-informe.

El segundo objetivo radica en comprobar cómo afecta a los resultados el orden de presentación de las categorías de respuesta en una pregunta sobre los sentimientos que suscitan las víctimas.

Partiendo de estos objetivos, se plantean las siguientes hipótesis:

$\mathrm{H}_{1}$ : Los encuestados pensarán en las víctimas de los delitos más graves, a pesar de que éstos son los menos representados en las estadísticas delictivas oficiales.

$\mathrm{H}_{2}$ : Los encuestados pensarán en las víctimas de los delitos que se producen con mayor frecuencia según las estadísticas delictivas oficiales.

$\mathrm{H}_{3}$ : Los encuestados, al tratarse de un cuestionario auto-administrado, tenderán a elegir las primeras opciones de respuesta de entre las mostradas (efecto primacía).

\section{Método}

\subsection{Diseño de investigación e instrumento}

El estudio se ha desarrollado de acuerdo a un diseño split-ballot. Este diseño experimental responde a un procedimiento que comporta dividir la muestra en dos mitades equivalentes, administrándose un cuestionario ligeramente diferente a cada una de ellas. La asignación de los sujetos a cada una de las mitades se ha efectuado mediante un criterio de procedimiento aleatorio dentro de los distintos grupos de edad. De tal forma que, para cada grupo de edad, los sujetos se han asignado aleatoriamente a cada uno de los grupos (Figura 1).

Revista Española de Investigación Criminológica

Artículo 6, Número 16 (2018)

www.criminologia.net

ISSN: 1696-9219 


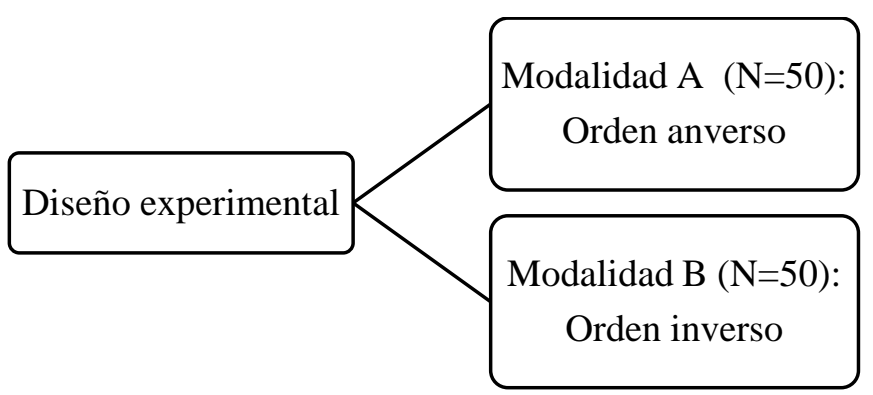

Figura 1. Diseño del experimento split-ballot

Los datos se recogieron a través de dos modalidades de un mismo cuestionario más amplio elaborado ad hoc para conocer la opinión de los ciudadanos respecto a temas relacionados con la justicia penal en España. Una de las preguntas se mantuvo inalterable en ambas modalidades de cuestionario y se presentó de forma abierta, de modo que los encuestados podían responder con sus propias palabras "Ahora nos gustaría que imagine a la víctima de un delito. ¿En qué tipo de delito/s piensa?’. La pregunta sobre los sentimientos que suscitan las víctimas de un delito se formuló de forma cerrada, balanceando las opciones de respuesta para que hubiese atributos positivos y negativos. La manipulación experimental consistió en la modificación del orden de presentación de las categorías de respuesta, de modo que el orden en la modalidad B era inverso al orden presentado en la modalidad A (Tabla 1).

\section{Tabla 1.}

Variación en la pregunta sobre sentimientos que suscitan las víctimas en ambas modalidades

\begin{tabular}{|c|c|}
\hline Modalidad A & Modalidad B \\
\hline $\begin{array}{l}\text { ¿Y podría decirme, de los siguientes, qué } \\
\text { sentimientos le provoca a usted } \\
\text { personalmente la víctima de un delito? (Por } \\
\text { favor, seleccione hasta un máximo de tres } \\
\text { respuestas) }\end{array}$ & $\begin{array}{l}\text { ¿Y podría decirme, de los siguientes, qué } \\
\text { sentimientos le provoca a usted } \\
\text { personalmente la víctima de un delito? (Por } \\
\text { favor, seleccione hasta un máximo de tres } \\
\text { respuestas) }\end{array}$ \\
\hline Culpabilidad.. & Incredulidad..... \\
\hline Valentía......... & Entereza........ \\
\hline Superación..................................... 3 & Tristeza....................................... 3 \\
\hline Indignación................................. 4 & Compasión.......................................... 4 \\
\hline Fortaleza.................................... 5 & Fortaleza...................................... 5 \\
\hline
\end{tabular}




\begin{tabular}{|c|c|}
\hline .... 6 & Indignación.... \\
\hline Tristeza...... & Super \\
\hline Entereza.... & Valentía....... \\
\hline Incredulidad.. & Culpabilidad... \\
\hline
\end{tabular}

\subsection{Participantes}

El tamaño muestral fue de 100 sujetos residentes en España. Las edades de los participantes estuvieron comprendidas entre los 19 y los 90 años, siendo su media de edad 45 años (DT= 20.48). El 52.0\% fueron mujeres y el $48.0 \%$ hombres. Sus principales características sociodemográficas desagregadas por modalidades se presentan en la Tabla 2.

\section{Tabla 2.}

Características sociodemográficas por grupos

\begin{tabular}{|c|c|c|}
\hline & $\begin{array}{c}\text { Modalidad A: } \\
\text { orden anverso } \\
\%(\mathbf{n})\end{array}$ & $\begin{array}{c}\text { Modalidad B: } \\
\text { orden inverso } \\
\%(n)\end{array}$ \\
\hline Edad (SD) $(t[99]=56,067, p=, 397)$ & $45.5(19.46)$ & $45.0(21.65)$ \\
\hline \multicolumn{3}{|l|}{$\operatorname{Sexo}\left(X^{2}[2]=2,003, p=, 157\right)$} \\
\hline Mujer & $59.2(29)$ & $44.9(22)$ \\
\hline Hombre & $40.8(20)$ & $55.1(27)$ \\
\hline \multicolumn{3}{|l|}{ Nivel educativo $\left(X^{2}[2]=5,526, p=, 596\right)$} \\
\hline Secundaria, Primaria o sin estudios & $10.2(5)$ & $10.2(5)$ \\
\hline Bachillerato o FP & $53.0(26)$ & $44.9(22)$ \\
\hline Graduado o más & $36.8(18)$ & $44.9(22)$ \\
\hline \multicolumn{3}{|l|}{ Ingresos $\left(X^{2}[2]=19,929, p=, 030\right)$} \\
\hline$\leq 900 €$ & $17.8(8)$ & $17.8(8)$ \\
\hline $901 €-3000 €$ & $64.4(29)$ & $68.9(31)$ \\
\hline$>3000 €$ & $17.8(8)$ & $13.3(6)$ \\
\hline
\end{tabular}

Nota: Las frecuencias no suman 100 debido a la existencia de "no respuesta" en algunas preguntas sociodemográficas.

La muestra se extrajo por conveniencia, con aplicación de cuotas de edad (18-30, 3150, 51-70 y 71 o más), distribuyéndose el número de participantes de manera proporcional entre los distintos grupos. 


\subsection{Procedimiento}

Una vez elaborado el instrumento de medida y antes de proceder a la recogida de los datos, se realizó una prueba piloto del cuestionario $(\mathrm{N}=30)$ para comprobar su claridad y comprensión. Los datos utilizados en este estudio se recogieron mediante encuesta autoadministrada en papel (PAP, Paper-and-Pencil), empleando para su cumplimentación una duración media de 18 minutos. Los cuestionarios fueron administrados durante los meses de enero a marzo de 2017 a diversos grupos sociales, procurando, con ello, reforzar la heterogeneidad de la muestra.

\subsection{Análisis de datos}

\subsubsection{Etapa 1. Codificación cualitativa de las respuestas a la pregunta sobre delitos}

En un primer momento se llevó a cabo la codificación de las respuestas recogidas en la pregunta sobre el tipo de delitos en los que piensan los encuestados al ser preguntados por las víctimas de un delito en general. Este proceso consistió en la creación de una serie de categorías más amplias y subcategorías más concretas a partir de la lectura de las respuestas recogidas en la encuesta (Richards, 2009; Silverman, 2011). Tras el análisis de las respuestas se conformaron 13 categorías y 28 subcategorías que incluyeron las diferentes tipologías delictivas enumeradas por los encuestados. A continuación se muestra un ejemplo de categoría y subcategorías creadas:

\section{Tabla 3.}

Ejemplo de categorías y subcategorías creadas

\begin{tabular}{|l|l|}
\hline \multirow{2}{*}{$\begin{array}{c}\text { Delitos contra la vida e } \\
\text { integridad física y moral }\end{array}$} & 1. Homicidio y asesinato \\
\cline { 2 - 2 } & 2. Lesiones y agresión \\
\cline { 2 - 2 } & 3. Maltrato \\
\cline { 2 - 2 } & 4. Amenazas \\
\hline
\end{tabular}




\subsubsection{Etapa 2. Análisis cuantitativo de los datos}

Una vez codificados los datos cualitativos se llevó a cabo el procesamiento y tratamiento estadístico de los datos. Para ello se utilizó el programa IBM-SPSS-Statistics en su versión 22.0. Se analizaron las distribuciones de frecuencias de cada una de las categorías para ambas preguntas y modalidades de cuestionario y se realizaron comparaciones de porcentajes y medias. Para ello, y en atención a las características de las variables, se utilizaron los estadísticos chi-cuadrado de Pearson y $t$ de Student. Los tamaños de efecto se calcularon mediante la $\mathrm{V}$ de Cramer.

\section{Resultados}

\section{1. ¿En qué delitos piensan los encuestados al ser preguntados por víctimas?}

En primera instancia se analizaron los delitos en los que piensan los encuestados al ser preguntados por las víctimas de un delito en abstracto. Como se muestra en la Figura 2, los resultados revelaron que los delitos que fueron enumerados con mayor frecuencia fueron el robo (73.5\%), el homicidio y el asesinato (28.6\%), la violación (23.5\%), la violencia de género $(18.4 \%)$ y las lesiones y agresiones (17.3\%). Tomados en conjunto, estos resultados indican que los encuestados piensan con mayor frecuencia en aquellos delitos más habituales (robo y lesiones). Sin embargo, también muestran en parte que, al igual que ocurre al preguntar por los delitos en los que piensan los encuestados cuando responden a preguntas relacionadas con los infractores (Cullen, Fisher, \& Applegate, 2000; Hough \& Roberts, 2002), existe cierta tendencia a considerar los hechos más graves cuando se formulan preguntas generales relacionadas con las víctimas. Y todo ello a pesar de que, como muestra la Tabla 4, estos delitos son los menos representados en las estadísticas oficiales (Ministerio del Interior, 2015) y en las encuestas de victimización (Díez-Ripollés \& García, 2009).

Este fenómeno ha sido ampliamente estudiado en la investigación criminológica, donde, entre otras variables explicativas tales como el daño social que producen estas victimizaciones o la especial sensibilidad ante los signos de violencia extrema, se ha destacado el poder que tienen los medios de comunicación para situar un determinado asunto 
en primer plano con independencia de la realidad del problema (Varona, 2011). En este sentido, Soto (2005) señalaba que "mientras que en las noticias de prensa los sucesos con mayor presencia cuantitativa son los homicidios y asesinatos, que representan cerca de un tercio del total de noticias sobre delincuencia, según las estadísticas policiales estos delitos alcanzan tan sólo el 0,06 de los hechos conocidos" (p.37).

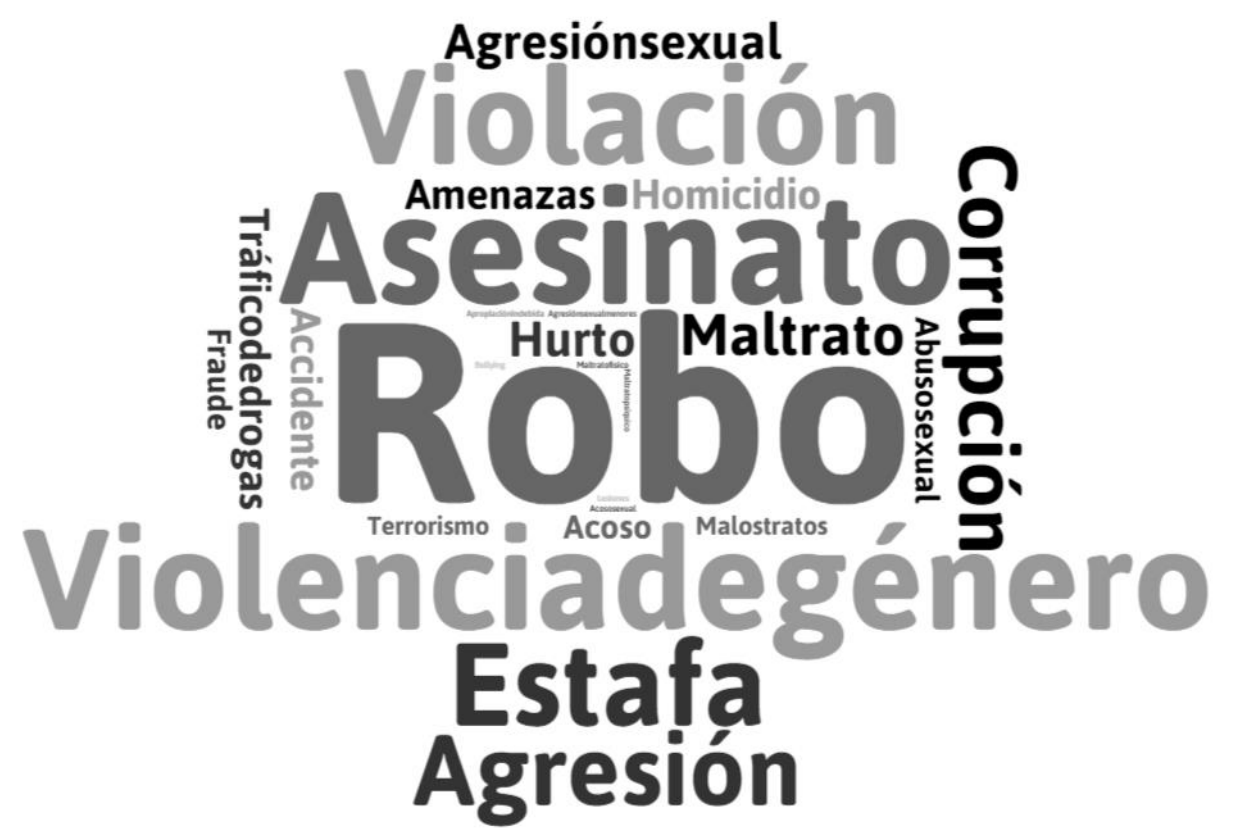

Figura 2. Nube de palabras de los delitos citados

Nota: El tamaño de las palabras representa la frecuencia con la que fueron citados.

No obstante, y como se planteó en la segunda hipótesis, los encuestados también citaron aquellos delitos que, según las estadísticas oficiales, ocurren con mayor frecuencia como son el robo y las lesiones (ver Tabla 4). Futuros estudios podrán profundizar en la posible relación entre el miedo a ser víctima de un delito y/o las experiencias previas de victimización y la probabilidad de enumerar una u otra tipología delictiva, dando respuesta a los siguientes interrogantes: ¿Mencionan los participantes aquellos delitos de los que tienen más miedo a convertirse en víctima?; ¿Citan aquellos delitos de los que han sido directa o indirectamente víctimas? 
Por otro lado, los delitos que fueron enumerados en menor proporción fueron los delitos contra la libertad de expresión (1.0\%), los delitos de odio (1.0\%), el delito de especulación (1.0\%), la represión sindical (1.0\%) y los delitos en la red (1.0\%). Además, en este sentido, resulta relevante que el delito de hurto fuese citado sustancialmente menos que el delito de robo a pesar de que, como muestran las estadísticas oficiales y de auto-informe, el primero ocurre con mayor frecuencia que el segundo (ver Tabla 4). No obstante, esto podría deberse a que la ciudadanía no tenga muy claros los elementos del tipo que conforman uno y otro delito, confundiendo las denominaciones.

Otro aspecto destacable es que la violencia de género, que hasta hace pocos años se consideraba un asunto privado y no estaba en el debate público, fuese uno de los delitos en los que se pensó con mayor frecuencia (18.4\%), ya que pone de relieve que los esfuerzos de sensibilización sobre la población están funcionando.

\section{Tabla 4.}

Comparación de las tipologías delictivas enunciadas con las estadísticas oficiales y los datos de auto-informe

\begin{tabular}{|l|c|c|c|}
\hline Tipo delictivo & $\boldsymbol{\%}^{\mathbf{a}}$ & $\begin{array}{c}\text { Estadísticas } \\
\text { oficiales }^{\mathbf{b}}\end{array}$ & $\begin{array}{c}\text { Estadísticas } \\
\text { auto-informe }^{\mathbf{c}} \%\end{array}$ \\
\hline Robo & 73.5 & 19.01 & 1.1 \\
\hline Homicidio y asesinato & 28.6 & 0.01 & - \\
\hline Violación & 23.5 & 0.06 & 0.2 \\
\hline Violencia de género & 18.4 & 0.76 & - \\
\hline Lesiones y Agresión & 17.3 & 4.76 & - \\
\hline Estafa & 17.3 & 8.11 & - \\
\hline Maltrato & 13.3 & - & 3.8 \\
\hline Corrupción & 10.2 & 0.03 & 0.2 \\
\hline Hurto & 8.2 & 35.12 & - \\
\hline Abuso sexual/Agresión sexual/ Acoso sexual & 7.1 & 0.37 & - \\
\hline Amenazas & 6.1 & - & - \\
\hline Acoso y bullying & 6.1 & - & - \\
\hline Tráfico de drogas & 5.1 & 0.59 & - \\
\hline Abuso sexual/Agresión sexual a menores & 4.1 & 0.06 & - \\
\hline
\end{tabular}


León \& Aizpurúa

\begin{tabular}{|c|c|c|c|}
\hline Tipo delictivo & $\%^{a}$ & $\begin{array}{l}\text { Estadísticas } \\
\text { oficiales }^{\text {b } \%}\end{array}$ & $\begin{array}{c}\text { Estadísticas } \\
\text { auto-informe } \%\end{array}$ \\
\hline Fraude & 3.1 & - & - \\
\hline Terrorismo & 3.1 & - & - \\
\hline Homicidio como resultado de un accidente & 3.1 & - & - \\
\hline Apropiación indebida & 2.0 & - & - \\
\hline Incendios provocados & 2.0 & - & - \\
\hline Abuso policial & 2.0 & - & - \\
\hline Abuso de poder & 2.0 & - & - \\
\hline Atropello & 2.0 & - & - \\
\hline Infracción de tráfico & 2.0 & 2.26 & - \\
\hline Violación de la libertad de expresión & 1.0 & - & - \\
\hline Delitos de odio & 1.0 & - & - \\
\hline Especulación & 1.0 & - & - \\
\hline Represión sindical & 1.0 & - & - \\
\hline Delitos en la red & 1.0 & - & - \\
\hline
\end{tabular}

${ }^{a}$ Los porcentajes no suman $100.0 \%$ porque los encuestados podían indicar múltiples delitos.

${ }^{\mathrm{b}}$ Elaborada a partir de los datos del Ministerio del Interior (2015).

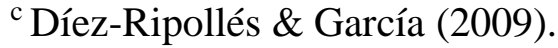

\subsection{En general, ¿piensan los encuestados en uno o en más delitos?}

En un segundo momento se analizó, por un lado, el número de delitos que fueron citados por los encuestados con el objetivo de conocer si éstos piensan en uno o en más delitos al responder a preguntas generales sobre víctimas y, por otro lado, se examinó si existían diferencias en el número de delitos enumerados por mujeres y hombres.

Como se muestra en la Figura 3, el número de encuestados que citó varios delitos fue muy superior (74.0\%) al número de encuestados que no enumeró ningún delito (2.0\%) o que sólo citó uno $(24.0 \%)$. Estos resultados podrían venir explicados por la influencia que tiene en los encuestados el tamaño del campo de respuesta en cuestionarios auto-administrados como este. De este modo, cuando el encuestado responde a preguntas abiertas, la tendencia es a rellenar el espacio facilitado para la respuesta. Así, cuanto mayor es el espacio, más extensa suele ser la respuesta (Christian \& Dillman, 2004; Maloshonok \& Terentev, 2016). 
Futuros estudios podrán profundizar en este aspecto aplicando un diseño split-ballot donde cada grupo de encuestados reciba un tamaño diferente de campo de texto.

Si atendemos a las diferencias por sexos, como se muestra en la Figura 3, el número de hombres y mujeres que citaron ninguno o un delito fue similar (27.6\% versus $23.5 \%)$. De igual modo, el número de mujeres y hombres que enumeraron varios delitos fue equivalente (76.5\% versus $72.3 \%$ ). Además, tras aplicar una prueba t para muestras independientes, los resultados confirmaron que no existieron diferencias significativas $(t[96]=-0.68, p>.05)$. En promedio, los hombres citaron aproximadamente el mismo número de delitos que las mujeres $(M=2.70$ versus $M=2.51)$.

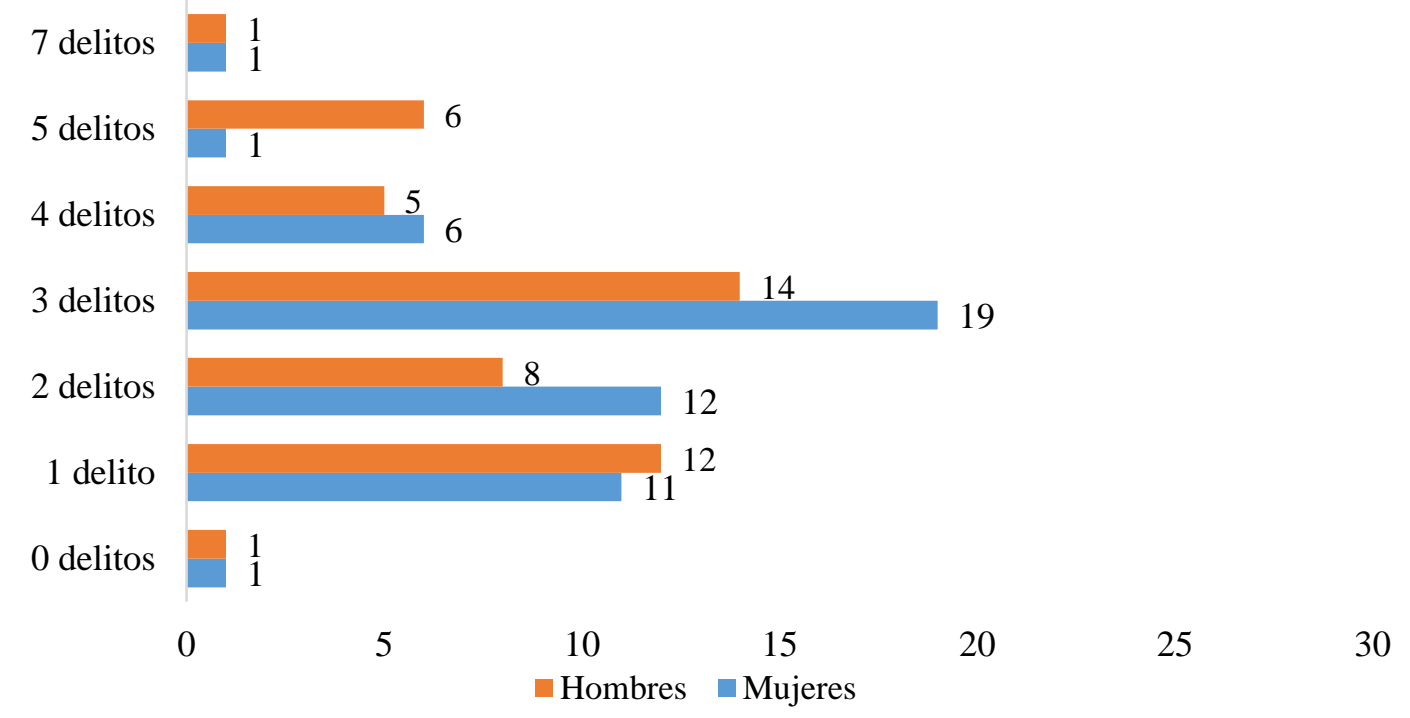

Figura 3. Número de delitos citados por hombres y mujeres (frecuencia)

\section{3. ¿Piensan mujeres y hombres en los mismos tipos de delitos?}

Con la finalidad de conocer si mujeres y hombres piensan en los mismos tipos delictivos al responder a preguntas generales sobre víctimas, se llevó a cabo un análisis pormenorizado de los delitos enumerados por uno y otro grupo. Como se muestra en la Tabla 5, solo fueron halladas diferencias significativas entre grupos en el delito de amenazas $\left(X^{2}[1]=5.88 ; p=.01\right.$; $V=.24)$. En este sentido, todas las veces que se enumeró este tipo delictivo fue citado por mujeres. También fueron halladas diferencias marginalmente significativas en los delitos de 
violencia de género $\left(X^{2}[1]=3.12 ; p=.07 ; V=.18\right)$ y homicidio como resultado de un accidente $\left(X^{2}[1]=2.84 ; p=.09 ; V=.09\right)$.

Además, se pueden destacar dos aspectos. En primer término, que las mujeres citaron en mayor medida que los hombres los delitos de maltrato, acoso y violación (ver Tabla 5). Atendiendo a las estadísticas oficiales, se observa que las mujeres están sobrerrepresentadas en estos tipos delictivos. En este sentido, considerando los datos facilitados por el Ministerio del Interior (2015), se encuentra que el número de mujeres victimizadas por malos tratos en el ámbito familiar es seis veces mayor al de hombres (50.500 versus 7.910). Lo mismo ocurre con la violación, donde la cifra de mujeres victimizadas es 10 veces mayor a la de hombres victimizados (827 en comparación con 82). En consecuencia, que las mujeres piensen más en estos tipos delictivos podría estar indicando, por un lado, que son conocedoras de que, por su condición de mujer, tienen más probabilidades de sufrir un delito de esta índole y, por otro lado, que tienen un mayor miedo a ser víctimas de estos delitos. Estos resultados vendrían avalados por estudios previos que han mostrado que las mujeres puntúan más alto en miedo al delito, en general, y en miedo a delitos sexuales, en particular. Además, la evidencia previa también manifiesta que las mujeres puntúan más elevado que los hombres en la percepción del riesgo de convertirse en víctimas (Fisher \& Sloan, 2006; Fox, Nobles, \& Piquero, 2009). En contraposición, los hombres enumeraron en mayor medida los delitos de estafa, fraude, terrorismo y corrupción (Tabla 5). Destaca en este sentido que la mayoría de estos delitos sean de índole económica.

En segundo término, resulta relevante que el delito de violencia de género fuera citado en mayor medida por los hombres que por las mujeres (66.7\% vs. 33.6\%). Estos resultados podrían estar mostrando que los esfuerzos de sensibilización están funcionando y que los hombres son cada vez más conscientes de esta lacra social.

\section{Tabla 5.}

Porcentajes de las categorías delictivas por sexo

\begin{tabular}{|l|c|c|c|c|}
\hline Tipo delictivo & Mujeres (N) & Hombres (N) & $\boldsymbol{X}^{2}$ & $\boldsymbol{p}$ \\
\hline Robo & 35 & 35 & 0.44 & 0.50 \\
\hline
\end{tabular}




\begin{tabular}{|c|c|c|c|c|}
\hline Tipo delictivo & Mujeres (N) & Hombres (N) & $X^{2}$ & $p$ \\
\hline Homicidio y asesinato & 16 & 12 & 0.40 & 0.52 \\
\hline Violación & 14 & 8 & 1.52 & 0.21 \\
\hline Violencia de género & 6 & 12 & 3.12 & 0.07 \\
\hline Lesiones y agresión & 8 & 8 & 0.06 & 0.85 \\
\hline Estafa & 7 & 10 & 0.98 & 0.32 \\
\hline Maltrato & 9 & 4 & 1.77 & 0.18 \\
\hline Corrupción & 3 & 7 & 2.18 & 0.14 \\
\hline Hurto & 4 & 4 & 0.01 & 0.90 \\
\hline Abuso sexual/ Agresión sexual/ Acoso sexual & 4 & 3 & 0.07 & 0.78 \\
\hline Amenazas & 6 & 0 & 5.88 & 0.01 \\
\hline Acoso y bullying & 4 & 2 & 0.54 & 46.00 \\
\hline Tráfico de drogas & 2 & 3 & 0.30 & 0.57 \\
\hline Abuso sexual/Agresión sexual a menores & 2 & 2 & 0.00 & 0.93 \\
\hline Fraude & 1 & 2 & 0.43 & 0.50 \\
\hline Terrorismo & 1 & 2 & 0.43 & 0.50 \\
\hline Homicidio como resultado de un accidente & 3 & 0 & 2.84 & 0.09 \\
\hline Apropiación indebida & 0 & 2 & 2.22 & 0.13 \\
\hline Incendios provocados & 0 & 2 & 2.22 & 0.13 \\
\hline Abuso policial & 0 & 2 & 2.20 & 0.13 \\
\hline Abuso de poder & 0 & 2 & 2.20 & 0.13 \\
\hline Atropello & 1 & 0 & 0.93 & 0.33 \\
\hline Infracción de tráfico & 1 & 1 & 0.00 & 0.95 \\
\hline Violación de la libertad de expresión & 0 & 1 & 1.09 & 0.29 \\
\hline Delitos de odio & 0 & 1 & 1.09 & 0.29 \\
\hline Especulación & 1 & 0 & 0.93 & 0.33 \\
\hline Represión sindical & 0 & 1 & 1.09 & 0.29 \\
\hline Delitos en la red & 0 & 1 & 1.09 & 0.29 \\
\hline
\end{tabular}

\section{4. ¿Afecta el orden de presentación de las categorías de respuesta a las respuestas de los encuestados?}


Por último, y con el objetivo de contrastar la tercera hipótesis planteada, se analizaron las distribuciones de las respuestas en la modalidad A (orden anverso de las categorías de respuesta) y en la modalidad B (orden inverso). Como se muestra en la Tabla 6, los resultados no permiten confirmar que se produzca el efecto primacía en las respuestas de los encuestados. En este sentido, solo fueron halladas diferencias significativas entre los grupos en dos de las nueve categorías de respuesta: valentía y fortaleza, y diferencias marginalmente significativas en la categoría de respuesta indignación. 'Valentía' ocupaba la segunda posición en la modalidad A, donde fue seleccionada el 16.3\% de las ocasiones, y la penúltima en la modalidad $\mathrm{B}$, donde los encuestados la eligieron en el $4.1 \%$ de las $\operatorname{veces}\left(X^{2}[1]=4.00\right.$; $p=.04)$. En este caso, la diferencia sí parece indicar una mayor elección de esta alternativa cuando es colocada al comienzo de la lista. Sin embargo, 'fortaleza' ocupaba la misma posición en ambas modalidades de cuestionario (categoría intermedia), por lo que la diferencia entre los grupos $\left(X^{2}[1]=5.01 ; p=.02\right)$ no puede ser explicada por la manipulación en el orden de las categorías de respuesta. En sintonía con esto, los resultados para la categoría de respuesta 'indignación' vuelven a mostrar que las diferencias entre modalidades $\left(X^{2}[1]=3.20 ; p=.07\right)$ no pueden ser explicadas por el efecto primacía, ya que en este caso la diferencia parece indicar una mayor elección de esta alternativa cuando es colocada al final de la lista.

Así, y a pesar de que la mayoría de estudios han documentado el efecto primacía en cuestionarios auto-administrados (Bishop et al., 1988; Israel \& Taylor, 1990; Krosnick \& Alwin, 1987; Schwarz \& Bless, 1992), otros no lo han hecho (Chang \& Krosnick, 2009) o sólo lo han encontrado en determinadas preguntas (Galesic et al., 2008; Stern et al., 2007). El presente estudio se ubica en la segunda categoría al no confirmarse la tendencia de los encuestados a seleccionar las primeras opciones de respuesta a pesar de tratarse de un cuestionario auto-administrado. Algunas de las razones que podrían explicar estos resultados son:

1. En primer término, el tema. Al tratarse de preguntas sobre las víctimas de delitos y los sentimientos que suscitan, los encuestados podrían tener muy formada su opinión, realizando un procesamiento igual de cuidadoso de todas las categorías de respuesta y seleccionando aquellas que mejor reflejan su opinión. 
2. En segundo término, el interés de los encuestados en el tema. Uno de los factores que contribuyen a los efectos de orden de las opciones de respuesta es la motivación de los encuestados, traduciéndose el interés en el tema en una mayor motivación. En este sentido, el $64.0 \%$ de los encuestados declaró tener bastante o mucho interés en la temática, lo que podría afectar, por un lado, a una lectura más cuidadosa de todas las opciones de respuesta y, por otro lado, a una menor fatiga.

3. Por último, otra circunstancia que podría afectar a la ausencia de efecto primacía en este experimento son las propias características de los encuestados. En este sentido, la evidencia muestra que ciertos perfiles de encuestado podrían ser más proclives a estar influenciados por el efecto del orden de las opciones de respuesta que otros. Así, Krosnick y Alwin (1987) hallaron que los encuestados con niveles cognitivos menos complejos eran más propensos a ser influenciados por el orden de las categorías de respuesta que aquellos individuos con un nivel cognitivo más sofisticado. En el presente estudio, si bien no se midió la capacidad cognitiva, la mayoría de los encuestados tenían estudios secundarios o terciarios (aproximadamente el 90.0\% de la muestra), lo que podría disminuir este efecto. Futuros estudios podrán profundizar en estos resultados analizando las diferencias entre quienes muestran un mayor efecto primacía en sus respuestas y quienes no lo hacen.

\section{Tabla 6.}

Distribuciones de las categorías de respuestas por grupos

\begin{tabular}{|l|c|c|c|c|c|c|}
\hline \multicolumn{1}{|c|}{ Sentimiento } & Modalidad A & Modalidad B & $\begin{array}{c}\text { Diferencia } \\
\text { porcentual }\end{array}$ & $\boldsymbol{X}^{2}$ & $\boldsymbol{p}$ & $\begin{array}{c}\text { Vde } \\
\text { Cramer }\end{array}$ \\
\hline Culpabilidad & $8.2 \%$ & $2.0 \%$ & $6.2 \%$ & 1.89 & .16 & .13 \\
\hline Valentía & $16.3 \%$ & $4.1 \%$ & $12.2 \%$ & 4.00 & .04 & .20 \\
\hline Superación & $18.4 \%$ & $18.4 \%$ & $0.0 \%$ & 0.06 & .79 & .02 \\
\hline Indignación & $63.3 \%$ & $79.6 \%$ & $-16.3 \%$ & 3.20 & .07 & .18 \\
\hline Fortaleza & $18.4 \%$ & $4.1 \%$ & $14.3 \%$ & 5.01 & .02 & .22 \\
\hline Compasión & $40.8 \%$ & $46.9 \%$ & $-6.1 \%$ & 0.37 & .54 & .06 \\
\hline Tristeza & $55.1 \%$ & $61.2 \%$ & $-6.1 \%$ & 0.66 & .41 & .08 \\
\hline Entereza & $12.2 \%$ & $4.1 \%$ & $8.1 \%$ & 2.17 & .14 & .14 \\
\hline Incredulidad & $22.4 \%$ & $14.3 \%$ & $8.1 \%$ & 1.08 & .29 & .10 \\
\hline
\end{tabular}


Nota: Los porcentajes no suman $100.0 \%$ porque los encuestados podían escoger hasta tres respuestas.

Por otro lado, si se realiza un análisis detallado de las respuestas, los resultados muestran que los sentimientos que evocan las víctimas de delitos son, para ambos grupos de encuestados, mayoritariamente negativos ('indignación', 'compasión', 'tristeza' e 'incredulidad'). Especialmente resulta relevante que, para ambos grupos, las categorías de respuesta más seleccionadas fuesen ‘indignación', 'compasión' y 'tristeza'. Estos resultados podrían venir explicados por dos razones. En primer lugar, parece ineludible hacer mención a la llamada implicación o identificación espontánea con la víctima a través de la cual el miedo o el dolor experimentado por las víctimas se imaginan en uno mismo. Además, mediante el acercamiento a la víctima, se humaniza y comprende el sufrimiento del otro, lo que genera sentimientos de preocupación, compasión o pesadumbre por ellos (Fernández \& Revilla, 2016). Por otro lado y como ocurre al preguntar por los delitos en los que piensan los encuestados cuando responden a preguntas relacionadas con los infractores, al preguntar por los sentimientos que suscitan las víctimas de un delito en abstracto, la tendencia también podría ser a considerar aquellas víctimas que han sufrido un mayor daño. Además, el hecho de que la pregunta sobre sentimientos que generan las víctimas se encontrase a continuación de la pregunta sobre los delitos en los que piensa el encuestado cuando imagina a las víctimas de un delito podría haber influido en que los encuestados contestasen pensando en las víctimas de la primera pregunta que, como ha quedado reflejado, en un número importante de casos eran víctimas de aquellos delitos más graves (violación y homicidio).

Además, un $8.2 \%$ de los encuestados en la modalidad A y un $2.0 \%$ en la modalidad B indicaron que las víctimas les provocaban principalmente sentimientos de culpabilidad. Se considera que este aspecto es destacable porque pone de relieve cómo sigue existiendo una cierta tendencia a culpabilizar a las víctimas por los delitos ocurridos. Esto refuerza la necesidad de mantener los esfuerzos de sensibilización que se han venido haciendo para evitar las experiencias de re-victimización. Otro aspecto remarcable de los resultados es que la 'superación' fue el sentimiento positivo escogido en mayor medida en ambos grupos (18.4\% en ambas modalidades). Futuros estudios podrán profundizar en estos resultados,

\section{Revista Española de Investigación Criminológica}


analizando los sentimientos que evocan las distintas tipologías delictivas. También será de utilidad evaluar cómo influyen las características de los encuestados (experiencias de victimización, consumo mediático...) en las actitudes hacia las víctimas.

\section{Conclusiones}

Los resultados del estudio permiten trazar dos conclusiones principales. En primer lugar, se constata que los encuestados piensan mayoritariamente en aquellos delitos más frecuentes (robo y lesiones) al ser preguntados por las víctimas de delitos en abstracto. Sin embargo, y a pesar de que existe una diferencia porcentual importante, también demuestran que los encuestados piensan en aquellos delitos más graves (homicidio, asesinato y violación) al responder a este tipo de preguntas generales. Estos resultados, sin despreciar el impacto que pudiesen tener otras variables, podrían mostrar el efecto de los medios de comunicación en la sobredimensión del fenómeno de la delincuencia y, más concretamente, en la sobredimensión de aquellos delitos que responden a un patrón más grave de comportamiento. Futuros estudios podrán profundizar en este sentido analizando la posible relación entre el consumo de medios, la atención prestada a los mismos y la credibilidad que la ciudadanía les otorga con la probabilidad de enumerar unas u otras tipologías delictivas.

La segunda de las conclusiones que se deducen de este trabajo es que los resultados no permiten confirmar que se produzca el efecto primacía en la elección de las alternativas de respuesta a pesar de tratarse de un cuestionario auto-administrado. Las razones que podrían explicar estos resultados son diversas (la temática de la encuesta, el interés de los participantes en la misma o las propias características de los encuestados). Sin embargo, y debido al tamaño muestral, no se ha podido analizar la incidencia de estas variables en la ausencia de sesgo de primacía, lo cual intensifica la necesidad de avanzar en este sentido, explorando la incidencia de distintas variables y valorando la contribución de cada una de ellas a los resultados obtenidos.

Por último, es preciso señalar que, a pesar de sus aportaciones, los resultados de este estudio deben interpretarse con cautela debido a la utilización de un procedimiento de muestreo no aleatorio y a una muestra reducida. Sin embargo, suponen una contribución 
importante al ámbito de las actitudes hacia las víctimas de delitos, especialmente debido a la escasa literatura existente en España sobre este campo de estudio. 


\section{Referencias}

Anuario Estadístico del Ministerio del Interior de España (2015). Disponible en: http://www.interior.gob.es/documents/642317/1204854/Anuario-Estadistico2015.pdf/03be89e1-dd38-47a2-9ce8-ccdd74659741

Applegate, B., Cullen, F., Link, B., Richards, R., \& Lanza-Kaduce, L. (1996). Determinants of public punitiveness toward drunk driving: A factorial survey approach. Justice Quarterly, 13, 57-79. doi: 10.1080/07418829600092821

Ayidiya, S. A. \& McClendon, M. J. (1990). Response effects in mail surveys. Public Opinion Quarterly, 54, 229-247. doi: 10.1086/269200

Baz, O., Aizpurúa, E. \& Fernández, E. (2015). Factores explicativos de las actitudes hacia el castigo juvenil. Evidencias de un diseño factorial. Política y Sociedad, 52, 869-895. doi: 10.5209/rev_POSO.2015.v52.n3.48400

Bishop, G. F., Hipplet, H. J., Schwarz, N., \& Strack, F. (1988). A comparison of response effects in self-administered and telephone surveys. En R. Gorves, J. T. Lars Lyberg, W. L. Nichols, \& J. Waksberg (Eds.), Telephone Survey Methodology (pp.321-340). New York: Wiley.

Brocke, M., Goldenitz, C., Holling, H., \& Bilsky, W. (2004). Attitudes towards severity of punishment: A conjoint analytic approach. Psychology, Crime \& Law, 2, 205-219. doi: 10.1080/10683160310001614793

Cameron, C. A. \& Stritzke, W. G. (2003). Alcohol and acquaintance rape in Australia: Testing the presupposition model of attributions about responsibility and blame. Journal of Applied Social Psychology, 5, 983-1008. doi: 10.1111/j.15591816.2003.tb01935.x

Chang, L. \& Krosnick, J. A. (2009). National surveys via RDD telephone interviewing vs. the Internet: Comparing sample representativeness and response quality. Public Opinion Quarterly 73, 641-678. doi: 10.1093/poq/nfp075

Christian, L. M., \& Dillman, D. A. (2004). The influence of graphical and symbolic language manipulations on responses to self-administered questions. Public Opinion Quarterly, 68, 58-81. doi: 10.1093/poq/nfh004

Cullen, F. T., Fisher, B. S., \& Applegate, B. K. (2000). Public opinion about punishment and corrections. Crime and Justice, 27, 1-79. doi: 10.1086/652198

DeSantis, A. \& Kayson, W. A. (1997). Defendants' characteristics of attractiveness, race, and sex and sentencing decisions. Psychological Reports, 81, 679-683. doi: 10.2466/pr0.1997.81.2.679

Díez-Ripollés, J. L. \& García, E. (2009). Encuesta a víctimas en España. Málaga: Instituto Andaluz Interuniversitario de Criminología. 
Fernández, C. \& Revilla, J. C. (2016). Seres "humanos" o seres "lejanos": Imágenes de violencia real e implicación/distanciamiento con las víctimas. Comunication \& Society, 29, 103-118.

Fisher, B. S. \& Sloan, J. J. (2006). Campus crime: Legal, social \& policy perspectives. Springfield, IL: Charles C Thomas.

Fox, K. A., Nobles, M. R., \& Piquero, A. R. (2009). Gender, crime victimization and fear of crime. Security Journal, 22, 24-39. doi: 10.1057/sj.2008.13

Galesic, M., Tourangeau, R., Couper, M. P., \& Conrad, F. G. (2008). Eye-tracking data. New insights on response order effects and other cognitive shortcuts in survey responding. Public Opinion Quarterly, 5, 892-913. doi: 10.1093/poq/nfn059

Hills, A. M. \& Thomson, D. M. (1999). Should victim impact influence sentences? Understanding the community's justice reasoning. Behavioral Sciences \& the Law, 5, 661-671. doi: 10.1002/(SICI)1099-0798(199923)17:5<661::AIDBSL369>3.0.CO;2-N

Holbrook, A. L., Krosnick, J. A., Moore, D. \& Tourangeau, R. (2007). Response order effects in dichotomous categorical questions presented orally. The impact of question and respondent attributes. Public Opinion Quarterly, 71, 325-348. doi: 10.1093/opq/nfm024

Hough, M. \& Roberts, J. V. (2002). Public knowledge and public opinion of sentencing. In: Tata, C. \& Hutton, N. (Eds.): Sentencing and society: international perspectives. (pp. 272). New York: Ashgate.

Israel, G. D. \& Taylor, C. L. (1990). Can response order bias evaluations? Evaluation and Program Planning, 13, 365-371. doi: 10.1016/0149-7189(90)90021-N

Jerre, K. (2014). More sanctions-less prison? A research note on the severity of sanctions proposed by survey participants and how it is affected by the option to combine a prison term with other sanctions. European Journal on criminal Policy \& Research, 20, 121-136. doi: 10.1007/s10610-013-9215-5

Knaïper, B. (1999). The impact of age and education on response order effects in attitude measurement. Public Opinion Quarterly, 63, 347-370. doi: 10.1086/297724

Krosnick, J. A. (1991). Response strategies for coping with the cognitive demands of attitude measures in surveys. Applied Cognitive Psychology, 5, 213-236. doi: 10.1002/acp. 2350050305

Krosnick, J. A. \& Alwin, D. F. (1987). An evaluation of a cognitive theory of response-order effects in survey measurement. The Public Opinion Quarterly, 2, 201-219. doi: $10.1086 / 269029$

Kury, H. \& Ferdinand, T. N. (1999). Miedo al delito, tamaño de la población, salidas a la calle y actitudes hacia la policía. Resultados alemanes. Revista de Derecho Penal y Criminología, 3, 209-292.

Revista Española de Investigación Criminológica

Artículo 6, Número 16 (2018)

https://doi.org/10.46381/reic.v16i0.158

Www.criminologia.net

ISSN: 1696-9219 
Maloshonok, N. \& Terentev, E. (2016). The impact of visual design and response formats on data quality in a web survey of MOOC students. Computers in Human Behaviour, 62, 506-515. doi: 10.1016/j.chb.2016.04.025

Mazzela, R. \& Feingold, A. (1994). The effects of physical attractiveness, race, socioeconomic status, and gender of defendants and victims on judgments of mock jurors: A meta-analysis. Journal of Applied Social Psychology, 15, 1315-1338. doi: 10.1111/j.1559-1816.1994.tb01552.x

Myers, B. \& Greene, E. (2004). The prejudicial nature of victim impact statements: Implications for capital sentencing policy. Psychology, Public Policy, and Law, 10, 492-515. doi: 10.1037/1076-8971.10.4.492

Niemi, L. \& Young, L. (2014). Blaming the victim in the case of rape. Psychological Inquiry, 25, 230-233. doi: 10.1080/1047840X.2014.901127

Niemi, L. \& Young, L. (2016). Justice and the moral lexicon. Psychological Inquiry, 27, 5054. doi: 10.1080/1047840X.2016.1111122

Picket, J. T., Mancini, C., \& Mears, D. P. (2013). Vulnerable victims, monstrous offenders, and unmanageable risk: Explaining public opinion on the social control of sex crime. Criminology, 51, 729-759. doi: 10.1111/1745-9125.12018

Quinn, S. B. \& Belson, W. A. (1969). The effects of reversing the order of presentation of verbal rating scales in survey interviews. London: Survey Research Centre.

Richards, L. (2009). Handling qualitative data: A practical guide. London: SAGE.

Rini, R. (2015). Microaggression, macro harm. Los Angeles Times.

Roberts, J. V., Stalans, L. J., Indemaur, D., \& Hough, M. (2003). Penal populism and public opinion. Lessons from five countries. New York: Oxford University Press.

Schwarz, N. \& Bless, H. (1992). Assimilation and contrast in attitude measurement: An inclusion/exclusion model. En J. F. Sherry \& B. Stemthal (Eds.), Advances in consumer research (pp.72-77). Provo, UT: Association for Consumer Research.

Silverman, D. (2011). Interpreting qualitative data. London: SAGE.

Simonson, J. (2011). Problems in measuring punitiveness - Results from a German study. In: Kury, H., \& Shea, E. (Eds). Punitivity international developments. Vol. 1: punitiveness - A global phenomenon?(pp.277-302). Bochum: Universitätsverlag Brockmeyer.

Sjöberg, M. \& Sarwar, F. (2017). Who gets blamed for rapes: Immigration status on the attribution of blame toward victims and perpetrators. Journal of Interpersonal Violence, 1-18. doi: 10.1177/0886260517703371

Soto, S. (2005). La influencia de los medios en la percepción social de la delincuencia. Revista Electrónica de Ciencia Penal y Criminología, 9.

Revista Española de Investigación Criminológica

Artículo 6, Número 16 (2018)

https://doi.org/10.46381/reic.v16i0.158

Www.criminologia.net

ISSN: 1696-9219 
Steinberg, L. \& Piquero, A. R. (2010). Manipulating public opinion about trying juveniles as adults: An experimental study. Crime \& Delinquency, 56, 487-506. doi: $10.1177 / 0011128708330179$

Stern, M. J., Dillman, D. A., \& Smyth, J. D. (2007). Visual design, order effects, and respondent characteristics in a self-administered survey. Survey Research Methods, 1, 121-138. doi: 10.18148/srm/2007.v1i3.600

Tourangeau, R. (1984). Cognitive science and survey methods. In: Thomas, B. J., Miro, L. S., Tanur, J. M., Tourangeau, R. (Eds.): Cognitive aspects of survey design: Building a bridge between disciplines. (pp.73-100). Washington, DC: National Academy Press.

Varona, D. (2011). Medios de comunicación y punitivismo. InDret: Revista para el análisis del Derecho, 1, 1-35.

\section{Agradecimientos}

Queremos agradecer al investigador David Vázquez su colaboración en el trabajo de campo de esta investigación. También agradecemos al editor y a los revisores anónimos de la REIC las aportaciones realizadas que han contribuido a mejorar la calidad científica del trabajo.

Carmen María León es investigadora predoctoral en formación en el Centro de Investigación en Criminología de la Universidad de Castilla-La Mancha. Sus principales líneas de investigación son percepciones y actitudes hacia la justicia; género y victimización.

(iD) https://orcid.org/0000-0002-0591-6056

Eva Aizpurúa es investigadora postdoctoral en el Center for Social \& Behavioral Research de la Universidad de Northern Iowa. Sus principales líneas de investigación incluyen percepciones y actitudes hacia la justicia; género, delincuencia y victimización, y metodología de encuestas.

iD https://orcid.org/0000-0001-7045-5535 